Textures and Microstructures, Vol. 31, pp. 53-84 Reprints available directly from the publisher Photocopying permitted by license only
(C) 1998 OPA (Overseas Publishers Association) N.V Published by license under the Gordon and Breach Science Publishers imprint. Printed in India.

\title{
X-RAY STUDY OF ROLLED SINGLE CRYSTALS OF Ti-48\%Ni-2\%Fe ALLOY. PART I: TEXTURE DEVELOPMENT AND MECHANISMS OF PLASTIC DEFORMATION
}

\author{
YU. PERLOVICH ${ }^{\mathrm{a}}$, H.J. BUNGE ${ }^{\mathrm{b}, *}$, M. ISAENKOVA ${ }^{\mathrm{a}}$, \\ V. FESENKO ${ }^{\mathrm{a}}$ and R. RUSTAMOV ${ }^{\mathrm{a}}$ \\ ${ }^{a}$ Moscow Engineering Physics Institute, Kashirskoe shosse 31, Moscow, \\ 115409, Russia; ${ }^{\mathrm{b}}$ Department of Physical Metallurgy, TU Clausthal, Grosser \\ Bruch 23, Clausthal-Zellerfeld 38678, Germany
}

(Received 29 July 1998)

Rolled single crystals of the alloy $\mathrm{Ti}-48 \% \mathrm{Ni}-2 \% \mathrm{Fe}$ were studied by methods of $\mathrm{X}$-ray diffractometry using a PSD-technique. Single crystals, consisting of the phase B2, were rolled in 11 initial orientations at $350^{\circ} \mathrm{C}$ with deformation degrees up to $\sim 80 \%$. Texture development in single crystals under rolling was analyzed to determine the acting mechanisms of plastic deformation. Three stable orientations of single crystals were found: $\{011\}\langle 011\rangle,\{111\}\langle 011\rangle$ and $\{111\}\langle 112\rangle$. It was shown on the basis of texture data, that $\mathrm{Ti}-\mathrm{Ni}$ single crystals deform by means of combined action of slip in the systems $\{011\}\langle 001\rangle$ and twinning. For the first time, along with twinning in the known systems $\{114\}\langle 221\rangle$, twinning in the systems $\{118\}\langle 441\rangle$ was revealed. A number of concrete cases was considered to refine the contributions of slip and twinning into reorientation of single crystals depending on their initial orientations as well as to ascertain the interrelation of different mechanisms.

Keywords: Ti-Ni alloy; Single crystal; Rolling texture; Reorientation; Stable orientation; Slip system; Twinning system

\section{O INTRODUCTION}

Alloys of the system $\mathrm{Ti}-\mathrm{Ni}$ fall into the category of the most perspective materials having the shape memory property. Though numerous

\footnotetext{
* Corresponding author.
} 
variants exist to use these alloys practically, details of their shape memory mechanisms are studied yet insufficiently. In particular, it is necessary to refine the crystallographic parameters of plastic deformation mechanisms, which determine the texture character and the shape memory anisotropy of concrete products manufactured from these alloys.

In the given paper reorientation of the crystalline lattice in rolled single crystals of the alloy $\mathrm{Ti}-48 \% \mathrm{Ni}-2 \% \mathrm{Fe}$ is studied, principles of texture development in these single crystals are revealed, and plastic deformation mechanisms responsible for the observed reorientation are analyzed. Since rolling is one of the main methods to deform metal materials in the course of their technological treatment, experiments on rolling of single crystals are of special interest in order to obtain data about deformation structure development within a single crystalline constituent of the real polycrystal, i.e. within a separate grain with a concrete crystallographic orientation. Such experiments is the necessary stage by handling a problem of texture control, which occurs to have an essential significance as applied to products with shape memory property, being a method to increase their exploitation characteristics.

\section{EXPERIMENTAL TECHNIQUE}

Single crystals of the B2-phase were manufactured from the alloy $\mathrm{Ti}-$ $48 \% \mathrm{Ni}-2 \% \mathrm{Fe}$ using the Bridgman method. The phase $\mathrm{B} 2$ has the $\mathrm{BCC}$ crystalline lattice ordered by the mode $\mathrm{CsCl}$ (Shimizu and Tadaki, 1987). According to estimates on the basis of X-ray measurements, an internal misorientation of the original single crystals within a volume of several cubic millimeters ranges, on the average, up to $10^{\circ}$ and can be described as a result of lattice rotation around one of the axes $\langle 001\rangle$.

Plates of $7 \times 7 \times 3 \mathrm{~mm}$ in sizes were cut out of the original single crystals by the electric spark method. The crystallographic orientations of plates were varied over a wide range; the total number of different orientation was 11 . The plates were packed in steel envelopes by welding in an inert atmosphere and then rolled at a temperature of $350^{\circ} \mathrm{C}$. Rolling at this increased temperature allowed to avoid the deformation-induced phase transformation $\mathrm{B} 2 \rightarrow \mathbf{R}$. The total deformation degree was varied in the range from $10 \%$ to $\sim 80 \%$ by an average reduction per pass of $3 \%$.

The initial crystallographic orientations of the plates as well as their deformation degrees are indicated in Table $I$. The actual average initial 
TABLE I Rolling of Ti-Ni single crystals: initial orientations and reorientations

\begin{tabular}{|c|c|c|c|c|}
\hline $\begin{array}{l}\text { Sample } \\
\text { number }\end{array}$ & $\begin{array}{c}\text { Initial } \\
\text { orientation }\end{array}$ & $\varepsilon, \%$ & $\begin{array}{l}\text { Reorientation features, } \\
\quad \text { final orientation }\end{array}$ & $\begin{array}{l}\text { Figure } \\
\text { number }\end{array}$ \\
\hline $\begin{array}{l}1 \\
2\end{array}$ & $\{001\}\langle 001\rangle$ & $\begin{array}{l}15 \\
44\end{array}$ & $\begin{array}{l}\text { Scattering by opposite rotations about TD; } \\
\text { The pair } \sim\{551\}\langle 011\rangle\end{array}$ & $\begin{array}{l}1(a, b) \\
1(c, d)\end{array}$ \\
\hline 3 & $\{001\}\langle 011\rangle$ & 10 & $\begin{array}{l}\text { Scattering up to }\{112\}\langle 111\rangle \text { by opposite } \\
\text { rotations about TD; }\end{array}$ & $2(a, b)$ \\
\hline 4 & & 58 & The pair $\{111\}\langle 011\rangle$ & $2(c, d)$ \\
\hline 5 & $\{115\}\langle 011\rangle$ & 15 & Fragmentation of initial maxima & $3(a, b)$ \\
\hline $\begin{array}{l}6 \\
7\end{array}$ & $\{011\}\langle 001\rangle$ & $\begin{array}{l}10 \\
56\end{array}$ & $\begin{array}{l}\text { Fragmentation of initial maxima; } \\
\text { The single component }\{111\}\langle 011\rangle\end{array}$ & $\begin{array}{l}4(a, b) \\
4(c, d)\end{array}$ \\
\hline 8 & $\{011\}\langle 011\rangle$ & 10 & $\begin{array}{l}\text { Stability of the initial orientation; } \\
\text { Small lateral shift }\end{array}$ & $5(a, b)$ \\
\hline $\begin{array}{r}9 \\
10\end{array}$ & & $\begin{array}{l}45 \\
77\end{array}$ & & $\begin{array}{l}5(c, d) \\
5(e, f)\end{array}$ \\
\hline 11 & $\{012\}\langle 012\rangle$ & 15 & $\begin{array}{l}\text { Gradual rotation about TD to } \\
\{011\}\langle 011\rangle+\text { further rotation about } \\
\text { RD in the direction of }\{111\}\langle 011\rangle\end{array}$ & $6(a, b)$ \\
\hline 12 & & 55 & & $6(c, d)$ \\
\hline 13 & & 59 & & $6(e, f)$ \\
\hline 14 & $\{013\}\langle 123\rangle$ & 10 & Fragmentation of initial maxima & $7(a, b)$ \\
\hline 15 & $\{023\}\langle 023\rangle$ & 26 & $\begin{array}{l}\text { Gradual rotation to }\{111\}\langle 011\rangle \\
\text { accompanied by some scattering } \\
\text { of initial maxima }\end{array}$ & $8(a, b)$ \\
\hline 16 & & $\begin{array}{l}56 \\
63\end{array}$ & & $\begin{array}{l}8(c, d) \\
8(e, f)\end{array}$ \\
\hline 11 & & OS & & \\
\hline 18 & $\{113\}\langle 345\rangle$ & 43 & $\{111\}\langle 011\rangle+$ traces of rotation & $9(a, b)$ \\
\hline 19 & $\{111\}\langle 011\rangle$ & 39 & $\begin{array}{l}\text { Stability of the initial orientation+arising } \\
\text { of additional maxima at a distance of } 20^{\circ}\end{array}$ & $10(a, b)$ \\
\hline 20 & $\{111\}\langle 112\rangle$ & 66 & Some shift from the stable orientation & $11(a, b)$ \\
\hline
\end{tabular}

orientations of the single crystals correspond to indicated orientations with a precision of $2-3^{\circ}$.

An addition of $\mathrm{Fe}$ to the equiatomic Ti-Ni alloy decreases the temperature of the martensitic transformation $\mathrm{B} 2 \rightarrow \mathrm{R}$ (Agafonov et al., 1990), so that under conditions of the X-ray study all rolled samples remain single-phase.

The X-ray study of the rolled single crystals included:

(a) measurement of conventional pole figures $\{001\}$, $\{011\}$ and $\{112\}$, i.e. normalized distributions of integral intensity of the $\mathrm{X}$-ray lines (002), (011) and (112); 
(b) measurement of generalized pole figures for other main parameters of the same X-ray lines, in particular pole figures of line broadening (that is half-width) and pole figures of peak position.

While conventional pole figures describe the texture of the sample, generalized pole figures characterize its substructure and elastic microstress distribution. For the first time the pole figures of line broadening were constructed by Perlovich (1983) for rolled molybdenum, the pole figures of peak position by Hoffmann et al. (1983) for rolled steels; Wcislak and Bunge (1996) described measurement of the generalized pole figures as a routine procedure using the modern diffractometric technique.

In the present work the X-ray texture diffractometer SIEMENS D500/ TX with a position sensitive detector (PSD) was used. Measurements were carried out with $\mathrm{CuK}_{\alpha}$ radiation. The main stages of data treatment were described earlier (Perlovich et al., 1997). The angular radius of the obtained incomplete pole figures was equal to $70^{\circ}$.

The X-ray data obtained for rolled $\mathrm{Ti}-\mathrm{Ni}$ single crystals can be considered in different aspects, among which there are, in particular, the following:

(1) reorientation of single crystals under rolling, unstable and stable orientations, fragmentation in consequence of inhomogeneous reorientation, texture development;

(2) active micromechanisms of plastic deformation;

(3) structure inhomogeneity of the rolled crystals, as it is seen from pole figures of line broadening;

(4) elastic microstresses in the rolled crystals, as they are seen from peak position pole figures;

(5) correlation between local substructure parameters and elastic microstresses.

This first part of the paper deals with aspects (1) and (2), while other aspects will be considered in next parts.

\section{REORIENTATION OF SINGLE CRYSTALS UNDER ROLLING}

Pole figures $\{001\}$ and $\{011\}$ for the rolled single crystals are exhibited in Figs. 1-11. The ideal initial orientations of the crystals are shown by 


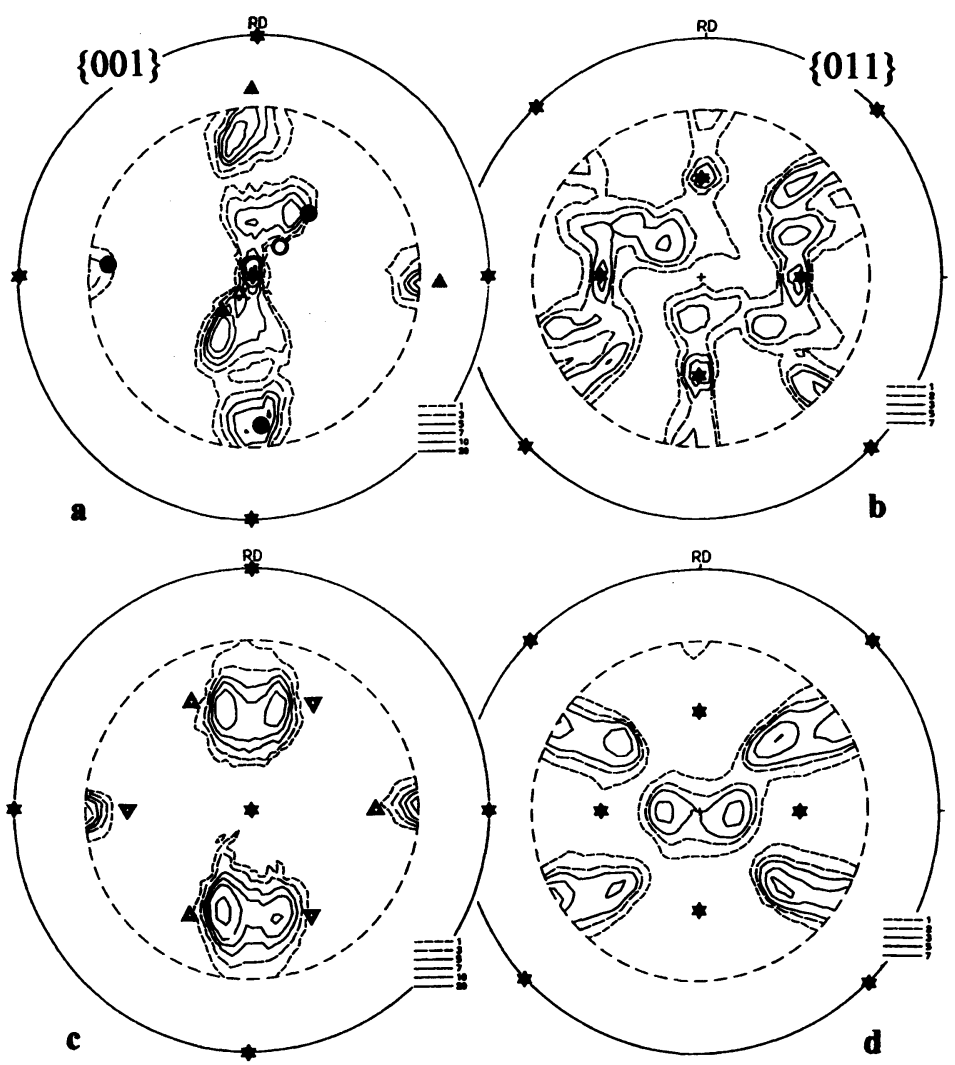

FIGURE 1 Pole figures $\{001\}(\mathrm{a}, \mathrm{c})$ and $\{011\}$ (b,d) for rolled single crystals with the initial orientation $\{001\}\langle 001\rangle$ after deformation degrees $15 \%(a, b)$ and $44 \%(c, d)$. Here and in the following figures the initial orientations are marked with the sign $(*)$ and final stable orientations $\{111\}\langle 011\rangle$ - with the sign $(\boldsymbol{A})$.

black stars. The main features of reorientation of the crystals under rolling are indicated in Table I. The following abbreviations are used: ND - normal direction (normal to the rolling plane), RD - rolling direction; TD - transverse direction.

The rolled crystals show the following different modes of reorientation:

(1) at the initial stages of deformation a single crystal breaks into several fragments in the orientational space, so that their maxima in the pole figures are mutually isolated or connected by zones of scattering; 


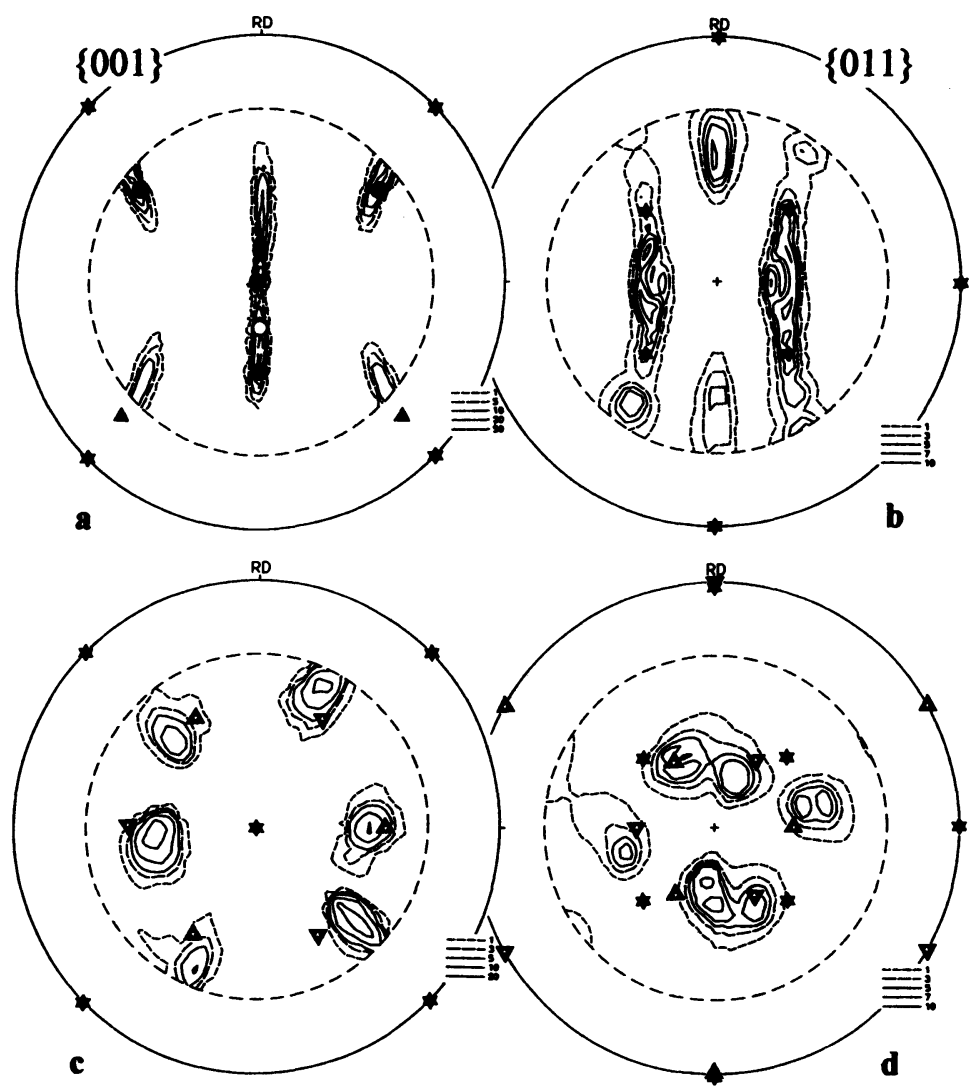

FIGURE 2 Pole figures $\{001\}(a, c)$ and $\{011\}$ (b,d) for rolled single crystals with the initial orientation $\{001\}\langle 011\rangle$ after deformation degrees $10 \%(a, b)$ and $58 \%(c, d)$.

(2) a single crystal reorients as a unit, without additional internal misorientation;

(3) a single crystal reorients gradually, so that some of its fragments move slower in the orientation space than its main body and, as a result, noticeable traces remain along the reorientation trajectory;

(4) there are two crystallographically equivalent ways of reorientation, so that a single crystal "can not" prefer any of them and, in consequence of their local competition, a significant scattering develops in opposite directions symmetric about TD or RD;

(5) an orientation does not change in the course of rolling either from the beginning of deformation, or from some intermediate stage; 

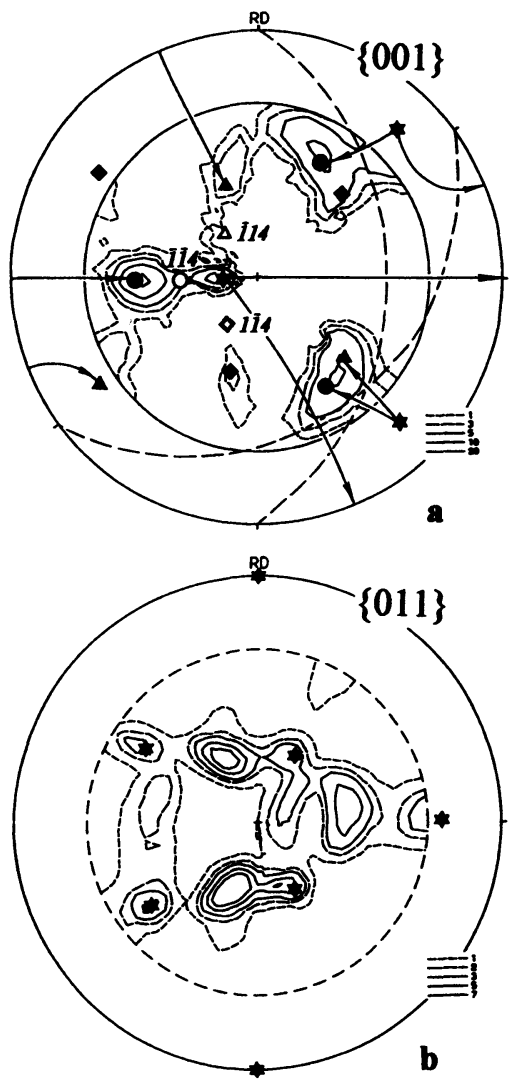

FIGURE 3 Pole figures $\{001\}$ (a) and $\{011\}$ (b) for the rolled single crystal with the initial orientation $\{115\}\langle 011\rangle$ after the deformation degree $15 \%$. The scheme of reorientation due to twinning by planes $\{114\}$ is shown; see necessary explanations in the text.

(6) scattering of the stable orientation increases gradually as the deformation degree grows.

As a rule, each reorientation mode makes itself evident only at definite stages of deformation depending on the initial orientation of a crystal. Sometimes different modes are observed simultaneously and, thus, manifest an inhomogeneity of the development of deformation in single crystals.

The kind of the acting mechanism of plastic deformation, that is slip or twinning, can be identified by the character of the resulting 

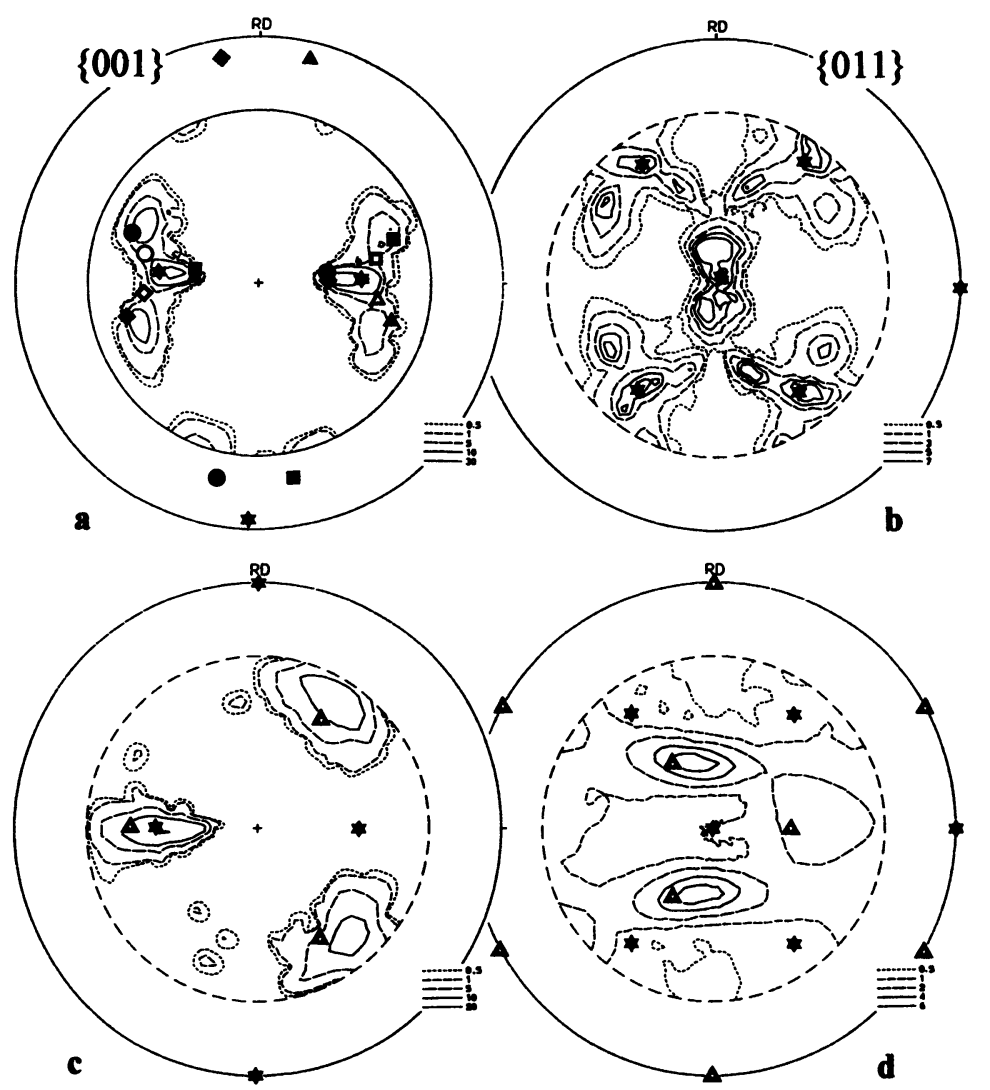

FIGURE 4 Pole figures $\{001\}(\mathrm{a}, \mathrm{c})$ and $\{011\}$ (b,d) for rolled single crystals with the initial orientation $\{011\}\langle 001\rangle$ after deformation degrees $10 \%(\mathrm{a}, \mathrm{b})$ and $56 \%(\mathrm{c}, \mathrm{d})$.

reorientation: slip causes continuous rotation of the crystalline lattice to its final stable orientation, whereas twinning results in jump-like lattice reorientation. Continuous movement of texture maxima in the pole figures or their breaking into separate fragments spaced at definite angles are corresponding manifestations of slip and twinning. The actual slip and twinning systems can be determined on the basis of models of texture formation by taking into account the directions of reorientation, stable orientations and the angular distances between texture maxima of initial and twinned components. 


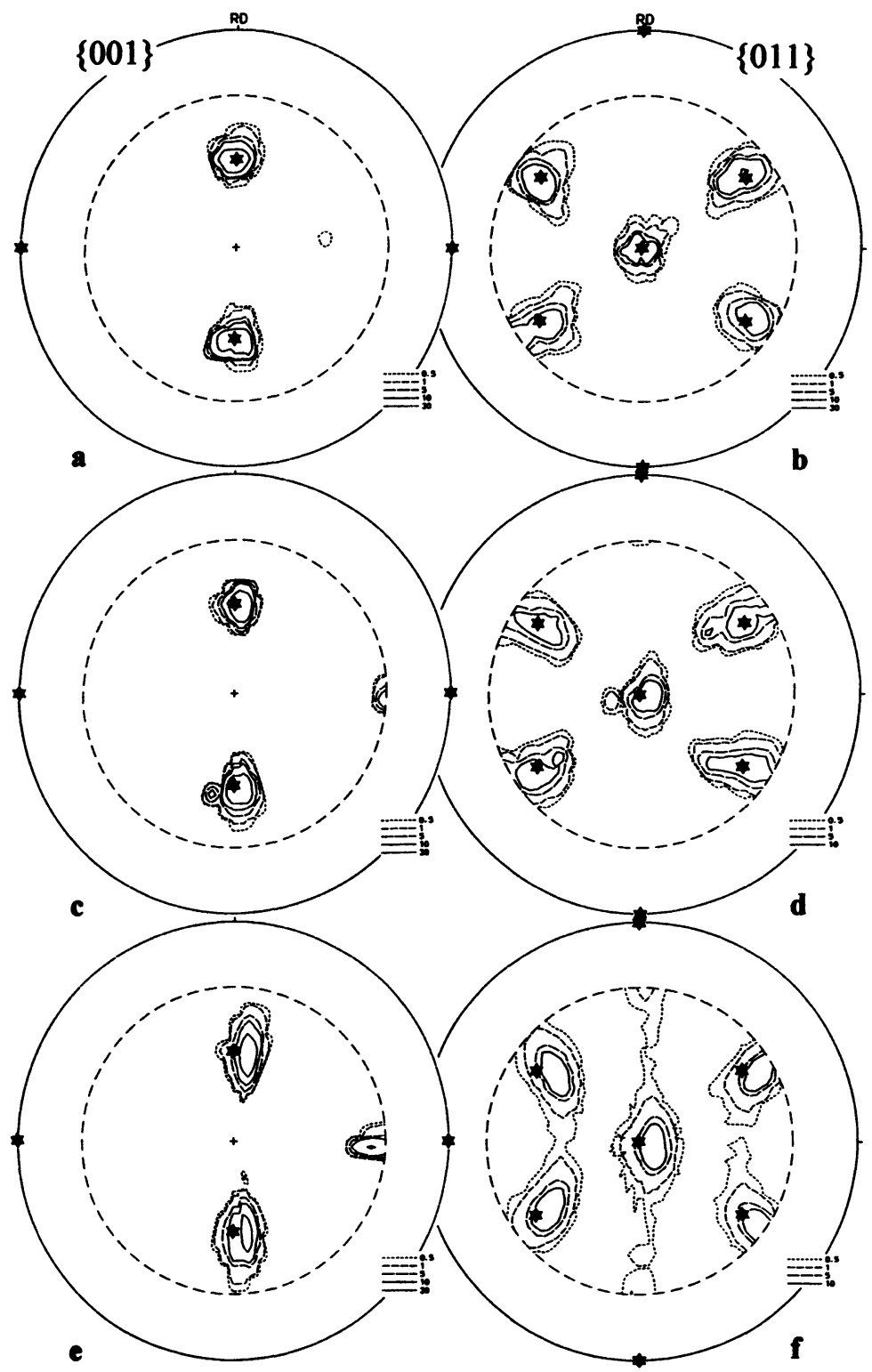

FIGURE 5 Pole figures $\{001\}(\mathrm{a}, \mathrm{c}, \mathrm{e})$ and $\{011\}$ (b,d,f) for rolled single crystals with the initial orientation $\{011\}\langle 011\rangle$ after deformation degrees $10 \%(a, b), 45 \%(c, d)$ and $77 \%(e, f)$. 


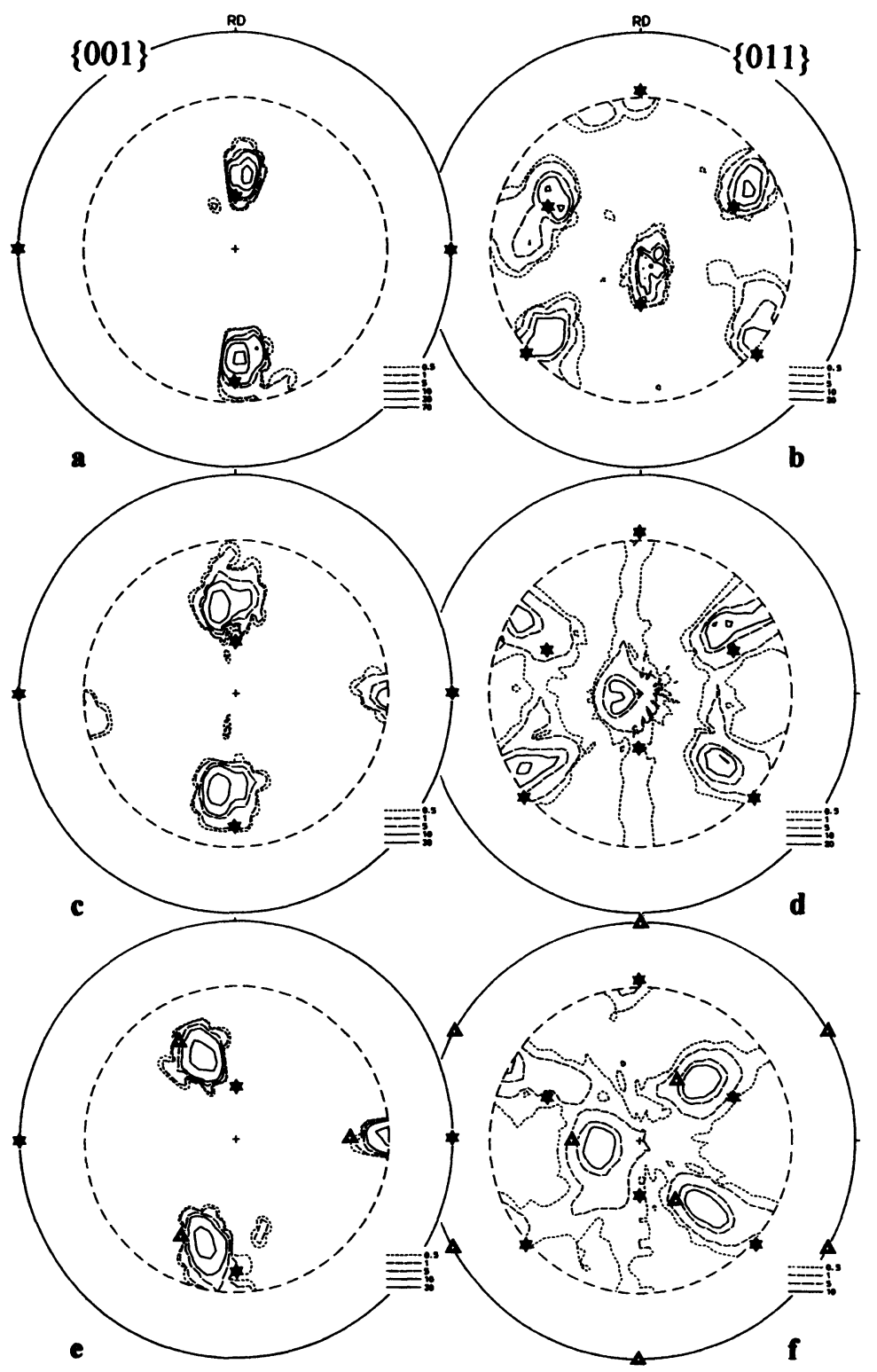

FIGURE 6 Pole figures $\{001\}$ (a,c,e) and $\{011\}$ (b,d,f) for rolled single crystals with the initial orientation $\{012\}\langle 012\rangle$ after deformation degrees $15 \%(a, b), 55 \%(c, d)$ and $59 \%(\mathrm{e}, \mathrm{f})$. 

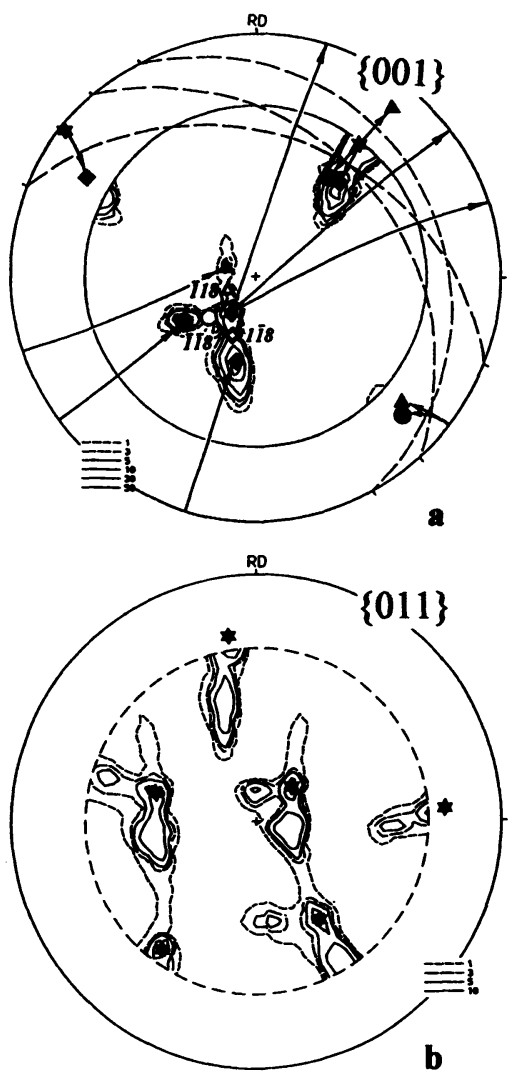

FIGURE 7 Pole figures $\{001\}$ (a) and $\{011\}$ (b) for the rolled single crystal with the initial orientation $\{013\}\langle 123\rangle$ after the deformation degree $10 \%$. The scheme of reorientation due to twinning by planes $\{118\}$ is shown; see necessary explanations in the text.

\section{MANIFESTATIONS OF THE SLIP SYSTEMS $\{011\}\langle 001\rangle$}

\subsection{Prediction of Reorientation Due to Slip by the Systems $\{011\}\langle 001\rangle$}

It is known, that in some intermetallic compounds (MgTl, $\mathrm{AuZn})$ with the crystalline lattice of the $\mathrm{CsCl}$ type the slip systems $\{011\}\langle 001\rangle$ are acting (Barrett and Massalski, 1966). For the B2-phase of Ti-Ni alloys there are also TEM observations of dislocations in planes $\{011\}$ with the Burgers vector parallel to $\langle 001\rangle$ (Moberly et al., 1990). Therefore, as the 


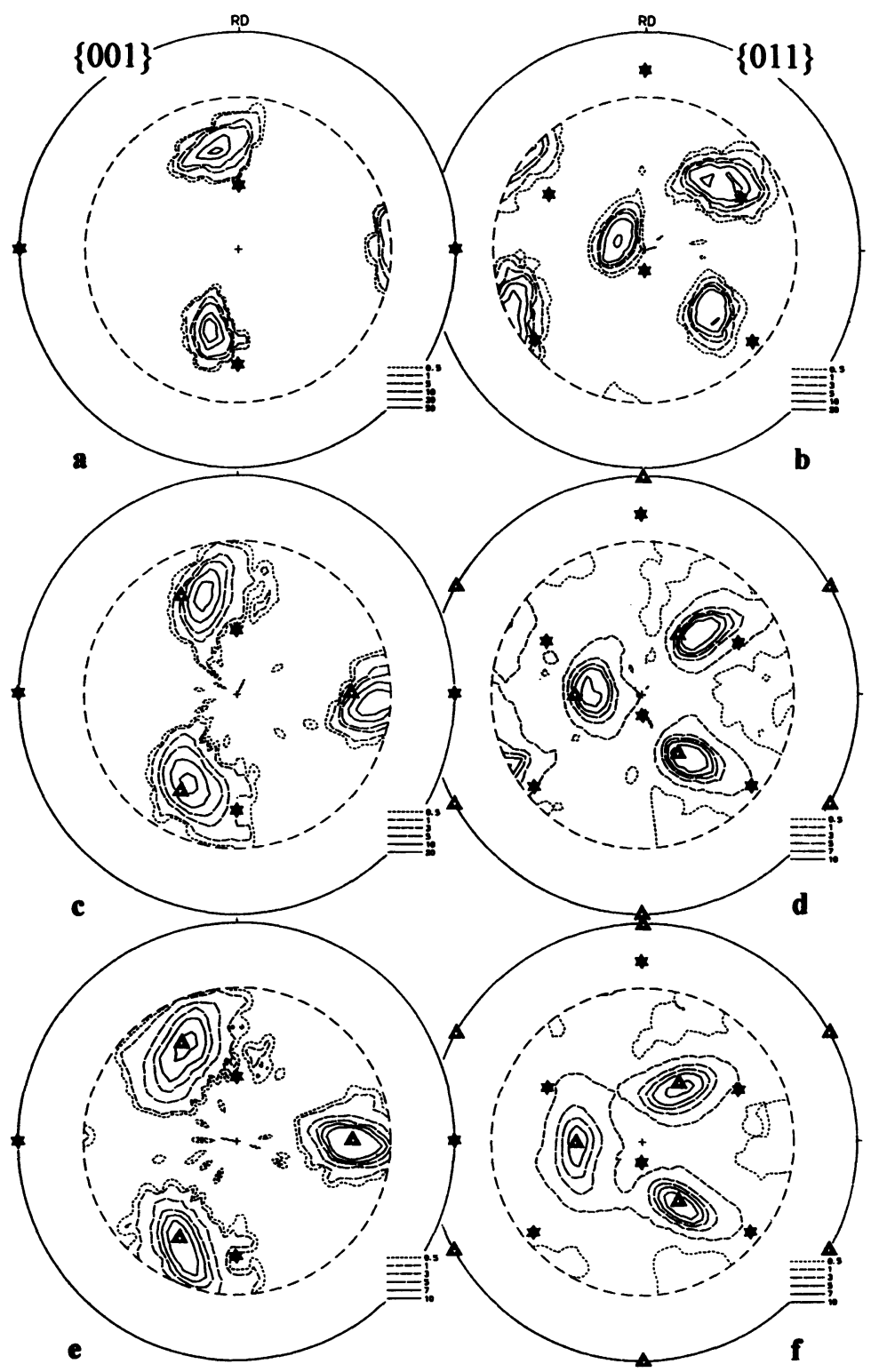

FIGURE 8 Pole figures $\{001\}$ (a,c,e) and $\{011\}$ (b,d,f) for rolled single crystals with the initial orientation $\{023\}\langle 023\rangle$ after deformation degrees $26 \%(a, b), 56 \%(c, d)$ and $63 \%(e, f)$. 

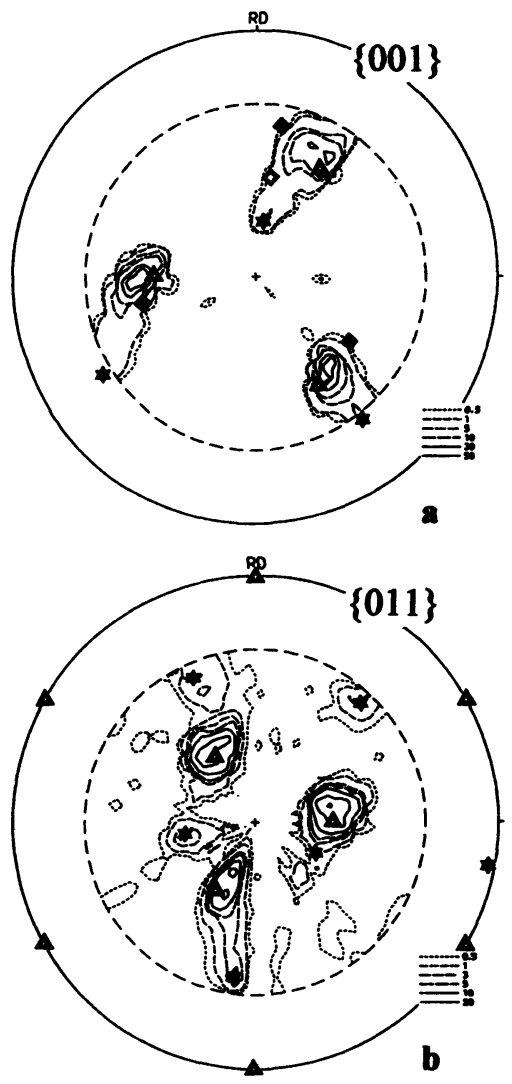

FIGURE 9 Pole figures $\{001\}$ (a) and $\{011\}$ (b) for the rolled single crystal with the initial orientation $\{113\}\langle 345\rangle$ after the deformation degree $43 \%$.

first step, it is worthwhile to consider to what extent the behavior of TiNi single crystals under rolling can be caused by action of the slip systems $\{011\}\langle 001\rangle$. In order to reveal, to what extent the observed reorientations of single crystals agree with the action of the slip systems $\{011\}\langle 001\rangle$, it is necessary first to calculate the distribution of the Schmid factor for these systems as well as to construct the expected trajectories of the loading axis in the orientational space. The latter can be done on the basis of models of texture formation.

In Fig. 12 the calculated distribution of the Schmid factor within a quadrant of the stereographic projection for three slip systems 

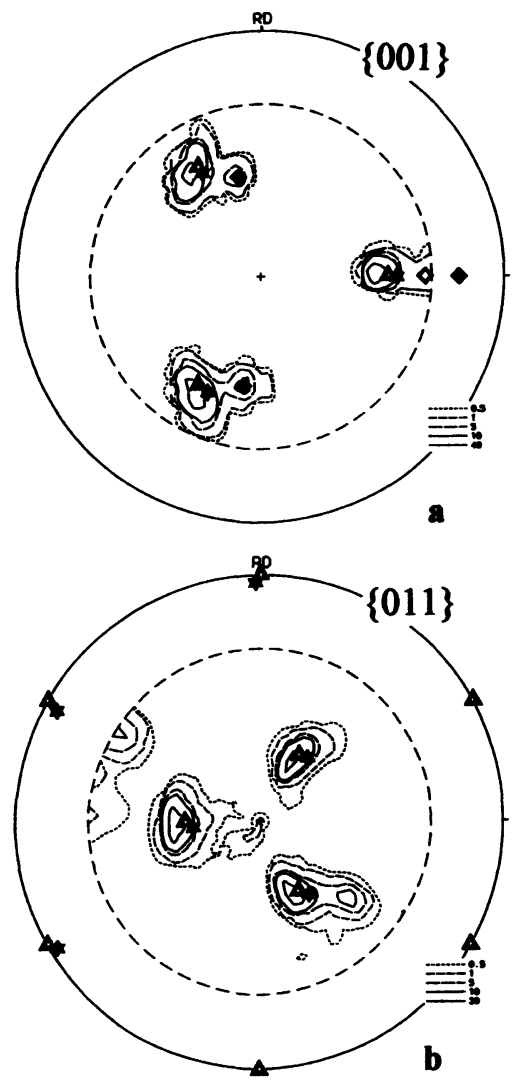

FIGURE 10 Pole figures $\{001\}$ (a) and $\{011\}$ (b) for the rolled single crystal with the initial orientation $\{111\}\langle 011\rangle$ after the deformation degree $39 \%$.

$\{011\}\langle 001\rangle$ is shown. The Schmid factor is the product $(\cos \lambda \times \cos \rho)$, where $\lambda$ and $\rho$ are angles between the loading axis and the normal to the slip plane and the slip direction, respectively. In Fig. 12 the Schmid factor for the slip system with maximal shear stress is plotted in each point. As it is seen from the distribution, when the loading axis is near $\langle 001\rangle$, shear stresses in slip systems $\{011\}\langle 001\rangle$ prove to be close to zero, so that deformation by means of these systems becomes impossible. In particular, the reorientation mode, which the single crystal would "choose" under rolling, seems to be most unpredictable in the case of the cubic initial orientation, when both compression and extension axes coincide 

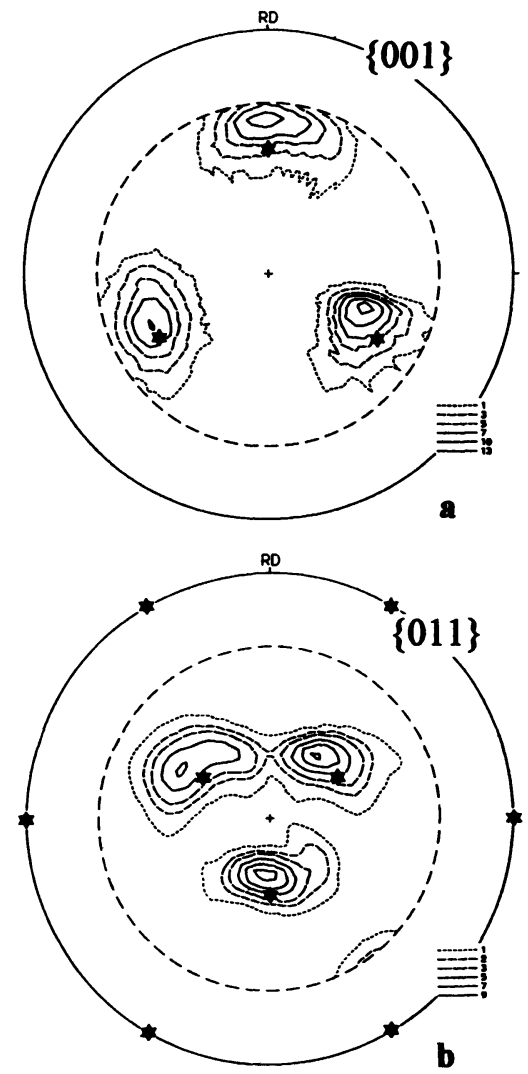

FIGURE 11 Pole figures $\{001\}$ (a) and $\{011\}$ (b) for the rolled single crystal with the initial orientation $\{111\}\langle 112\rangle$ after the deformation degree $66 \%$.

with $\langle 001\rangle$ directions. It is reasonable to expect, that in such cases twinning would be a mechanism, "throwing" the loading axis to a region of the stereographic triangle with higher values of the Schmid factor in slip systems. The obtained experimental data confirm the validity of this prediction, as will be shown somewhat further.

In order to construct the reorientation trajectories, the known principles of lattice rotation under uniaxial deformation (Calnan and Clews, 1950; Wassermann and Grewen, 1962) can be used. According to these principles, the crystalline lattice rotates in such a manner, that the axis of compression tends to align with the normal to the acting slip plane and 


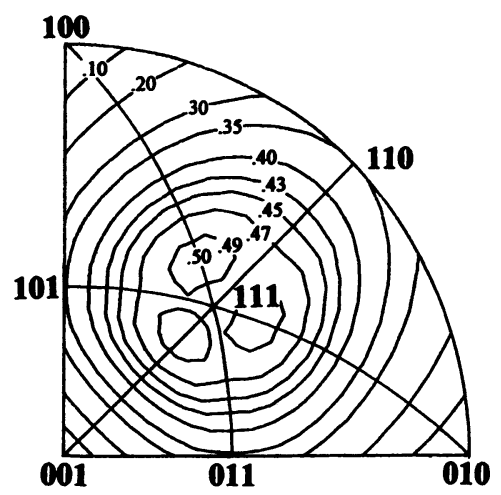

FIGURE 12 Calculated distribution of the Schmid factor for slip systems $\{011\}\langle 001\rangle$.

the axis of extension tends to align with the slip direction. However, both axes cannot attain their destination, since as the lattice rotates owing to action of the initially active slip system, the shear stress in the secondary slip system increases and its action becomes possible, too. Then the resulting movement of the loading axis is the geometric sum of the rotations due to both slip systems. As a result, the loading axis gets into a stable position, where its rotations due to different acting slip systems are mutually compensated.

Arrows in Fig. 13 indicate directions, along which the axes of compression (a) and extension (b) rotate by the action of the slip systems $\{011\}\langle 001\rangle$. Inside the stereographic triangle and along its boundary $\langle 001-111\rangle$ the loading axis reorients due to action of the slip system (110)[001], along the boundary $\langle 001-011\rangle$ due to action of systems (110)[001] and ( $\overline{1} 10)[001]$, along the boundary $\langle 011-111\rangle$ due to action of systems (110)[001] and (101)[010]. The length of the arrow corresponds to the shear stress in the active slip system for the case of the loading axis located at the point of interest. It is seen, that the compression axis moves towards the pole $\langle 111\rangle$, either directly, or past the pole $\langle 011\rangle$ along the boundaries $\langle 001-011-111\rangle$ of the triangle. Arrows of reorientation of the extension axis are directed in the opposite direction, that is towards the pole $\langle 001\rangle$. But actually, this pole cannot be attained, since within the middle region of the triangle the arrows become already too short, that is the shear stress in the acting slip system 
decreases strongly. As a result, the most probable final orientation of rolled single crystals by action of slip systems $\{011\}\langle 001\rangle$ is characterized by the rolling plane $\{111\}$ and the rolling direction between $\langle 011\rangle$ and $\langle 112\rangle$.

To a first approximation, rolling is described usually as a combination of compression along ND and extension along RD. It should be taken into account, that the deformation zone in rolling has a rather complicated character and includes regions of predominant compression and predominant extension, through which all portions of the sample are passing sequentially. Within each of these portions both compression and extension activate in turn their own slip systems, which cause the corresponding lattice rotations. Therefore, the prediction of reorientations of single crystals on the basis of the above models for uniaxial deformation seems to be warranted. At the same time, by the joint

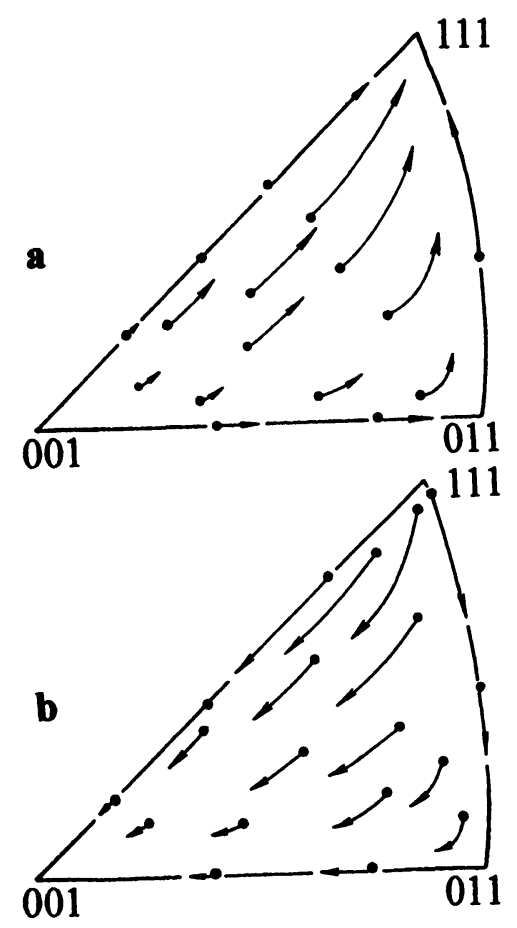

FIGURE 13 Predicted reorientations of loading axes due to slip by systems $\{011\}\langle 001\rangle$ : (a) axis of compression (ND); (b) axis of extension (RD). 
consideration of compression and extension, the systems of slip (or twinning) can be found, which are promoted by both loadings and therefore prove to be preferable. This criterion will be used further to estimate the probabilities of activation of different twinning systems.

\subsection{Cases of Obvious Slip in the Studied Single Crystals}

The experimental trajectories of compression and extension axes, that is ND and RD, under rolling of the studied crystals are presented in Fig. 14. They connect the initial orientations of the crystals with orientations, corresponding to main components of their rolling textures, and thus show only an average trend. According to the general tendency, these trajectories agree principally with the above prediction, so that the fact of action of slip systems $\{011\}\langle 001\rangle$ is at least not disproved. However, the drawn line between initial and final positions of loading axis depicts the real trajectory of this axis only in the case of its continuous reorientation by slip. If the loading axis passes from the initial position to the final one due to twinning, that is by a jump, the line between both positions is not a trajectory in the strict sense of the word. Hence, the action of slip systems $\{011\}\langle 001\rangle$ would be reliably confirmed only on the condition, that additional evidences exist in favor of the continuous character of observed reorientation.

Such character of reorientation is evident in the following cases:

(1) texture maxima move without accompanying scattering by relatively small steps, as it occurs with single crystals, having initial orientations $\{012\}\langle 012\rangle(N 11-13$, Fig. 6) and $\{023\}\langle 023\rangle(N 15-17$, Fig. 7);

(2) moving maxima leave behind them distinct continuous traces along their trajectories, as it is seen for the single crystal with the initial orientation $\{113\}\langle 345\rangle$ ( $N$ 18, Fig. 9);

(3) the rolling texture consists of a number of relatively small maxima, surrounded by an extended zone of continuous scattering, as it is seen in crystals with initial orientations of the loading axes near $\langle 001\rangle$ and, in particular, with initial orientations $\{001\}\langle 001\rangle(N 1$, Fig. 1(a) and (b)) and $\{001\}\langle 011\rangle(N 3$, Fig. 2(a) and (b)).

While in case (1) the crystals reorient as a unit, in case (2) reorientation develops inhomogeneously, so that some fraction of the single crystal 


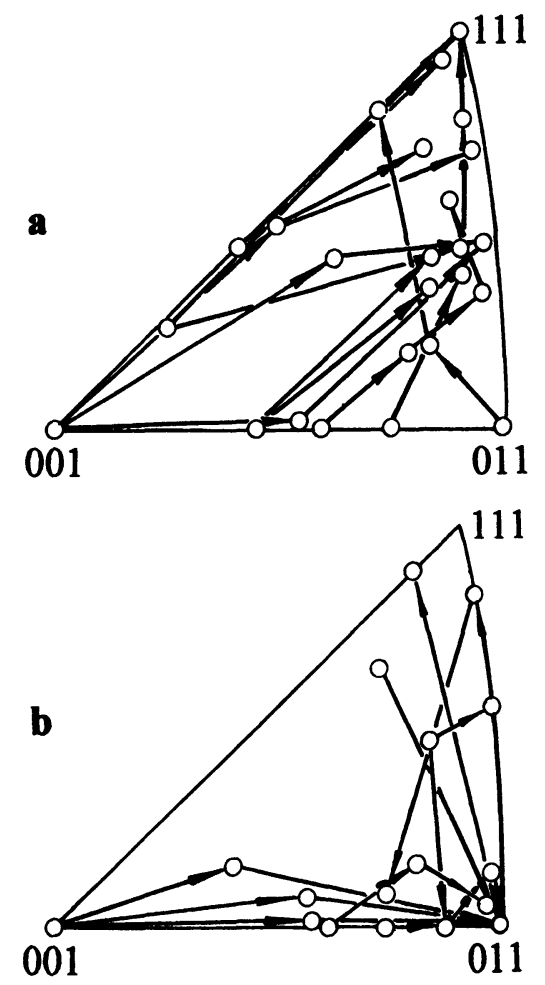

FIGURE 14 Experimental trajectories of compression (a) and extension (b) axes.

retains its initial orientation, though its most part has attained already the final orientation. This difference is connected, probably, with features of the corresponding trajectories: in case (1) ND moves along the boundaries $\langle 001-011-111\rangle$ of the elementary triangle owing to the action of two different slip systems $\{011\}\langle 001\rangle$, whereas in case (2) ND goes through the region of a single preferred slip system. It turns out that a pair of slip systems ensures a fuller reorientation, than a single system with a higher Schmid factor, which causes only a partial passage of the crystal to the new orientation.

The case (3) cannot be realized without participation of slip, since the occurrence of zones of continuous scattering requires small local rotations of the crystalline lattice. However, in this case slip is only a secondary mechanism of plastic deformation, whereas twinning proves to be, obviously, its primary mechanism. Activation of twinning promotes 
slip not only owing to a change of the initial orientation, but also owing to some interrelation of twinning and slip phenomena at the level of dislocation processes.

\subsection{Stable Orientations}

The existence of stable orientations is an additional evidence in favor of the action of slip by rolling of studied single crystals. There are three orientations of $\mathrm{Ti}-\mathrm{Ni}$ single crystals, showing stability under rolling:

- $\{011\}\langle 011\rangle$ is stable, at least, up to a deformation degree of $80 \%$, when being an initial orientation $(N 8-10$, Fig. 5$)$, and is metastable, i.e. transitory, when being attained as a result of rotation from an unstable orientation ( $N$ 12, Fig. 6);

- $\{111\}\langle 011\rangle$ proves to be stable as an initial orientation $(N 19$, Fig. 10$)$ as well as a final point for the majority of reorientation trajectories;

- $\{111\}\langle 112\rangle$ is rather stable, when being taken as an initial orientation (N 20, Fig. 11).

Orientation stability of the loading axis presumes the two conditions to be met:

(a) the single crystal deforms by several slip systems, each of which ratates the lattice in its own direction according to the abovementioned principles, so that different rotations compensate one another and, as a result, the crystal retains its orientation unchanged.

(b) the loading axis returns to the initial position after being deflected from it because of some mutual disbalance of the acting slip systems, connected with their local unequal strain hardening.

In principle, the condition (a) is obeyed certainly, when the loading axis coincides with any of the crystallographic directions $\langle 001\rangle,\langle 011\rangle$ or $\langle 111\rangle$, that is, when several mutually symmetric slip systems of the same type are acting in the single crystal. The condition (b) presumes, that the rotation of the loading axis is directed to the stable orientation in its vicinity, and requires the coincidence of the stable orientation with a minimum of the Schmid factor for the acting slip systems. As the loading axis moves away from the stable orientation because of braking of some slip system, the Schmid factor in this system increases, so that sooner or later the braked system reactivates repeatedly, resulting in the reverse movement of the loading axis. 
The orientation $\{011\}\langle 011\rangle$ satisfies the condition (b) not in a full measure, since the reorientation of ND along the boundary $\langle 011-111\rangle$ in Fig. 13(a) is directed away from the pole $\langle 011\rangle$. But the orientation $\{111\}\langle 011\rangle$ satisfies all necessary conditions of stability. Though, in the latter case, the orientation stability is expected to be most perfect, the scattering of texture maxima of the component $\{111\}\langle 011\rangle$ in all obtained pole figures is relatively strong. Texture maxima in pole figures of the single crystal, rolled in the orientation $\{111\}\langle 011\rangle$, even break into two submaxima, spaced at an angular distance of $\sim 20^{\circ}$ (Fig. 10(a)), which, as it will be shown further, corresponds to the reorientation by one of the twinning systems.

\section{TWINNING IN ROLLED SINGLE CRYSTALS}

\subsection{Twinning by the Systems $\{114\}\langle 221\rangle$}

The obtained texture data show clearly, that in many initial orientations of $\mathrm{Ti}-\mathrm{Ni}$ single crystals twinning proves to be the main mechanism of plastic deformation. In particular, in cases of splitting of the initial maxima into several new ones, we deal, surely, with manifestations of twinning. This mode of reorientation is observed only at the initial stages of rolling, with deformation degrees up to $10-15 \%$. Taking into account, that, according to TEM observations (Goo et al., 1985; Moberly et al., 1990), plastic deformation of TiNi occurs by a combination of dislocation slip and mechanical twinning, we can note the principal agreement of the data, obtained independently by TEM and X-ray texture analysis. The authors of the referred works notify that slip takes place in systems $\{011\}\langle 001\rangle$ and twinning - in systems $\{114\}\langle 122\rangle$. However, their TEM data concern polycrystalline material regardless of the orientation of the studied grains, whereas our X-ray data allow to connect the observed reorientation modes and corresponding deformation mechanisms with the initial orientation of the rolled single crystals.

A geometrical analysis of all obtained pole figures was undertaken in order to reveal, whether twinning could be really responsible for the concrete reorientation of the single crystal under rolling or not. The procedure of this analysis is known (Vishnyakov et al., 1979) and 
includes the following steps:

- in the pole figure $\{001\}$ (or $\{011\}$ ) of the rolled single crystal the initial orientations of normals $\langle 001\rangle$ (or $\langle 011\rangle$ ) as well as projections of all normals to probable twinning planes are marked;

- for the normal to each twinning plane the meridianal circle is drawn, that is a stereographic projection of this twinning plane;

- new twinned positions of the normals $\langle 001\rangle$ are found by symmetric carrying of their initial positions along parallels of the stereographic grid at the opposite side of the meridian, corresponding to the twinning plane of interest;

- if new positions of normals $\langle 001\rangle$ prove to be within maxima of the rolling texture, this argues that twinning by the corresponding plane controlled the process of reorientation.

The full procedure is shown schematically in Fig. 3(a) and 7(a) as applied to single crystals rolled in initial orientations $\{115\}\langle 011\rangle(N 5)$ and $\{013\}\langle 123\rangle(N$ 14), while in Fig. 1(a), 2(a) and 4(a) for rolled single crystals with initial orientations $\{001\}\langle 001\rangle(N 1),\{001\}\langle 011\rangle(N 3)$ and $\{011\}\langle 001\rangle(N 6)$ with deformation degrees of $10 \%$ or $15 \%$, only the orientations of normals to active twinning planes are indicated. These cases are the most obvious manifestations of twinning in the studied Ti-Ni single crystals. In all pole figures the initial orientations of the cubic axes are shown as black stars, normals to twinning planes as white squares, circles or triangles, and the twinned orientations of the cubic axes as black squares, circles or triangles, depending on the concrete active twinning plane. Projections of twinning planes in Fig. 3(a) and $7(a)$ are drawn as dashed meridians, while arrows connect initial and twinned orientations of cubic axes.

The analysis shows that in the single crystal with the initial orientation $\{115\}\langle 011\rangle$ there are three active twinning planes $\{114\}$ out of four possible ones, having their normals at the same angular distance from the normal $\langle 001\rangle$, situated initially in the vicinity of ND (Fig. 3(a)). Twinning does not develop in the fourth plane of the set, because its normal is too close to ND, so that the shear stress in the corresponding system of possible twinning is minimal. (The shear stress in a twinning system is determined in the same way, as the shear stress in a slip system.) It is evident also from the arrangement of normals to active twinning planes, that the choice of these planes is determined mainly by 
compression along ND rather than by extension along RD. For instance, the normal to the twinning plane, marked in Fig. 3(a) with the circle, is $90^{\circ}$ away from $R D$, so that the value of $\cos \lambda$ and the shear stress for this twinning system are zero. Nevertheless, comparison of all derivative maxima shows that just this twinning system was the most active under rolling of the single crystal.

\subsection{Twinning by the Systems $\{118\}\langle 441\rangle$}

The same analysis of pole figures for the rolled single crystal with the initial orientation $\{013\}\langle 123\rangle$ (Fig. $7(a)$ ) leads to the unambiguous conclusion, that its plastic deformation was due to twinning by planes $\{118\}$. The truth of this conclusion is clearly demonstrated by a simple geometrical construction in the PF $\{001\}$. So far we did not meet published communications about twinning in TiNi by planes $\{118\}$. While twinning by $\{114\}$ planes reorients the normals $\langle 001\rangle$ at an angular distance of $39^{\circ}$, twinning by $\{118\}$ planes reorients them at a distance of $21^{\circ}$ only. Though in single crystals with initial orientations $\{115\}\langle 011\rangle$ and $\{013\}\langle 123\rangle$ twinning occurs by planes of different types, that is $\{114\}$ and $\{118\}$ respectively, its development in both single crystals has a common feature, consisting in twinning by three planes out of four possible ones, the normals to which are equidistant from the pole $\langle 001\rangle$ in the central part of $\mathrm{PF}\{001\}$.

Twinning by planes $\{118\}$ forms likewise the rolling texture of the crystal, deformed by $10 \%$ in the initial orientation $\{011\}\langle 001\rangle$ (Fig. 4(a)). Four planes $\{118\}$ are active in this case: two of them have their normals near the left pole $\langle 001\rangle$ and two others near to the right pole $\langle 001\rangle$. Among the four normals $\langle 118\rangle$ in the vicinity of each pole $\langle 001\rangle$, those at the greater distance from ND belong to active planes. But it should be noted, that the coincidence of twinned orientations of cubic normals with maxima of the rolling texture is worse, than in the above-considered cases. This coincidence would be better, if it would be supposed that the actual twinning plane is deflected from $\{118\}$ inward of the elementary stereographic triangle. However, arguments in favor of the action of the additional twinning plane seem to be insufficient in this case, so that an alternative supposition, according to which the accompanying slip gives here also some contribution to plastic deformation and texture development, is more preferable. 
When comparing rolling textures of the single crystals with initial orientations $\{011\}\langle 001\rangle(N 6)$ and $\{011\}\langle 011\rangle(N 8)$, an effect of the initial rolling direction on the behavior of single crystals with the same initial rolling plane becomes visible: whereas the first crystal deforms by twinning accompanied by fragmentation of the initial maxima, the second one retains stability of its initial orientation, most probably, by means of balanced slip in symmetric systems.

The distribution of cubic axes for the rolled crystal with the initial orientation $\{001\}\langle 001\rangle(N$ 1, Fig. 1(a)) manifests distinctly, that in this case twinning is followed by intense slip, which causes an additional shift of the texture maxima away from the center of the pole figure. In Fig. 1(a) reorientations due to twinning by both planes $\{114\}$ and $\{118\}$ are shown at opposite sides of the central initial maxima. In particular, the normal $\langle 001\rangle$, located initially at the center of the pole figure, in consequence of twinning by the plane $\{118\}$, falls at the edge of the maximum below the center (a black triangle in Fig. 1(a)), whereas the subsequent shift of this normal due to the action of a slip system brings it inside the maximum. It should be noted, that an increase of the deformation degree up to $44 \%$ $(N$ 2, Fig. 1(c)) results in a further shift of the texture maxima in the direction of the orientation $\{111\}\langle 011\rangle$. Twinning by the plane $\{114\}$ (circles in Fig. 1(a)) requires a subsequent lateral shift of the maxima at a rather small angular distance in order to bring them into their actual positions. In this case, twinning is most likely to occur by systems of both types. Along with the main texture maxima, which are attributed to twinning by planes $\{114\}$ and $\{118\}$, the distribution of pole density shows a noticeable maximum at the diameter ND$\mathrm{RD}$, testifying about the possible action of some additional mechanism.

According to the rolling texture of the single crystal with the initial cubic orientation, among the four twinning planes $\{114\}$ or $\{118\}$, with normals in equivalent positions relative to both $\mathrm{ND}$ and $\mathrm{RD}$, only two of them are comparable in their activity. Since geometric reasons for this preference are absent, it should be concluded, that the observed asymmetry of $\mathrm{PF}\{001\}$ is connected with the real physical inhomogeneity of twinning within the single crystal.

One more variant of deformation by twinning is seen in the pole figures of the single crystal rolled in the initial orientation $\{001\}\langle 011\rangle$ with the deformation degree of $10 \%$ ( $N 3$, Fig. 2(a)). This crystal 
reorients in such a manner, that its rolling texture does not allow to establish, with certainty, the acting deformation mechanisms (slip or twinning). In principle, the scattering of the initial maxima by rotation about TD can be caused by slip. But taking into account the whole set of obtained data, it is more probable in this case to assume twinning. The scheme of reorientation in Fig. 2(a) confirms, that the extended texture maximum at the diameter ND-RD can be a result of simultaneous twinning by planes $\{114\}$ and $\{118\}$ with a predominance of the first. For simplicity, in order to avoid overlapping, twinning planes of different types are shown at opposite sides of the initial orientation. As in the previous case, also in the $\{001\}\langle 011\rangle$ crystal the participation of slip must be assumed. Obviously, twinning provides the necessary conditions for the activation of slip, though shear stresses in potential slip systems remain comparatively low.

The single crystal with the stable initial orientation $\{111\}\langle 011\rangle(N 19$, Fig. 10(a)) shows also evidence of some twinning. This consists in the emergence of additional small maxima at an angular distance of $\sim 20^{\circ}$ from the main ones, so that their positions correspond to twinning by one of the planes $\{118\}$. The orientation of the twinned fragment is rather close to its initial orientation $\{111\}\langle 011\rangle$ and, as a result, it proves to be stable as well. Though in this paper we do not take repeated twinning into account, it should be noted here, that in principle stability of the rolling texture can be obtained by the balanced continuous action of twinning and slip: while twinning throws the cubic normals to a distance of $20^{\circ}$ from their symmetric positions, the following slip returns them back.

Also it should be pointed out, that the above-considered behavior of the single crystal with the initial orientation $\{113\}\langle 345\rangle(N 18$, Fig. 9) can be interpreted in somewhat other manner, if twinning is assumed as a possible mechanism of reorientation. The twinning system $\{114\}\langle 221\rangle$ with the highest shear stress due to simultaneous loading along ND and RD is marked with a white rhombus in Fig. 9(a), whereas the twinned orientation of the normal $\langle 001\rangle$ is shown as a black rhombus. It can be seen, that the final orientation of this crystal can be attained by means of a jump due to twinning by the plane $\{114\}$, followed by some lateral shift due to slip by the system $\{011\}\langle 001\rangle$. In this variant the contribution of twinning and slip in plastic deformation is reversed compared to the first one. 


\subsection{Analysis of Reorientations by Use of the Schmid Factor for Twinning Systems}

For the sake of systematization of the presented data, concerning activation of different mechanisms, in addition to the Schmid factor for slip systems $\{011\}\langle 001\rangle$, also the Schmid factor for both observed systems of twinning was calculated. Since texture data do not carry an obvious information about the direction of twinning, by calculation of the Schmid factor for twinning by planes $\{114\}$, in accord with Goo et al. (1985), we took this direction to be $\langle 221\rangle$, whilst for twinning by planes $\{118\}$ the direction $\langle 441\rangle$ was assumed, so that the systems $\{114\}\langle 221\rangle$ and $\{118\}\langle 441\rangle$ are similar by their positions with reference to main crystallographic axes. In Fig. 15(b) a combined distribution of the Schmid factor is constructed for the twinning systems $\{114\}\langle 221\rangle$ and $\{118\}\langle 441\rangle$ as compared with the distribution for the slip systems $\{011\}\langle 001\rangle$ in Fig. 15(a). The stereographic triangle is divided into regions of preferable activation of specific twinning systems, so that, by location of the loading axis near to the normal $\langle 001\rangle$, the twinning system $\{114\}\langle 221\rangle$ is characterized by maximal values of the shear stress, whereas, by location of the loading axis between the normals $\langle 112\rangle$ and $\langle 011\rangle$, the shear stress is maximal in the twinning system $\{118\}\langle 441\rangle$.

Predictions of active twinning systems on the basis of the calculated distribution of the Schmid factor in Fig. 15(b) principally agree with the above-considered experimental data, though some discrepancies exist and require comments, which follow below.

(1) The single crystal rolled in the initial orientation $\{115\}\langle 011\rangle(N 5)$ shows predominant twinning by systems $\{114\}\langle 221\rangle$, whereas the single crystal with the initial orientation $\{013\}\langle 123\rangle(N 14)$ by systems $\{118\}\langle 441\rangle$. In both cases ND is situated within the region, corresponding to the preferential twinning by planes $\{114\}$, and RD within the region of twinning by planes $\{118\}$. The observed difference in behavior of these crystals under rolling is conditioned, evidently, by the difference in the values of the Schmid factor for the initial orientations of $\mathrm{RD}$, that is 0.35 for $\langle 011\rangle$ and 0.48 for $\langle 123\rangle$. As a result, the probability of twinning by $\{118\}$ planes, in the latter case, increases sharply.

(2) Along with the preferential twinning by $\{114\}$ planes, some evidence of twinning by planes $\{118\}$ is also seen in $\mathrm{PF}\{001\}$ for the rolled 
single crystal with the initial orientation $\{115\}\langle 011\rangle$ (Fig. 3). Hence, the critical shear stress for twinning by $\{118\}$ planes is lower, than for twinning by $\{114\}$ planes.

(3) The single crystal with the initial orientation $\{011\}\langle 001\rangle(N 6)$ has only a small calculated preference for twinning by planes $\{118\}$ owing to the position of ND. But the obtained experimental results show that actually this preference is stronger, than it can be predicted by calculation. This fact confirms the above conclusion about the lower critical shear stress for twinning by planes $\{118\}$.

(4) The calculated values of the Schmid factor for twinning systems in all regions of the triangle are very high, so that its value for the slip system proves to be lower almost everywhere, with the exception of a small region not far away from the pole $\langle 111\rangle$. Nevertheless, the

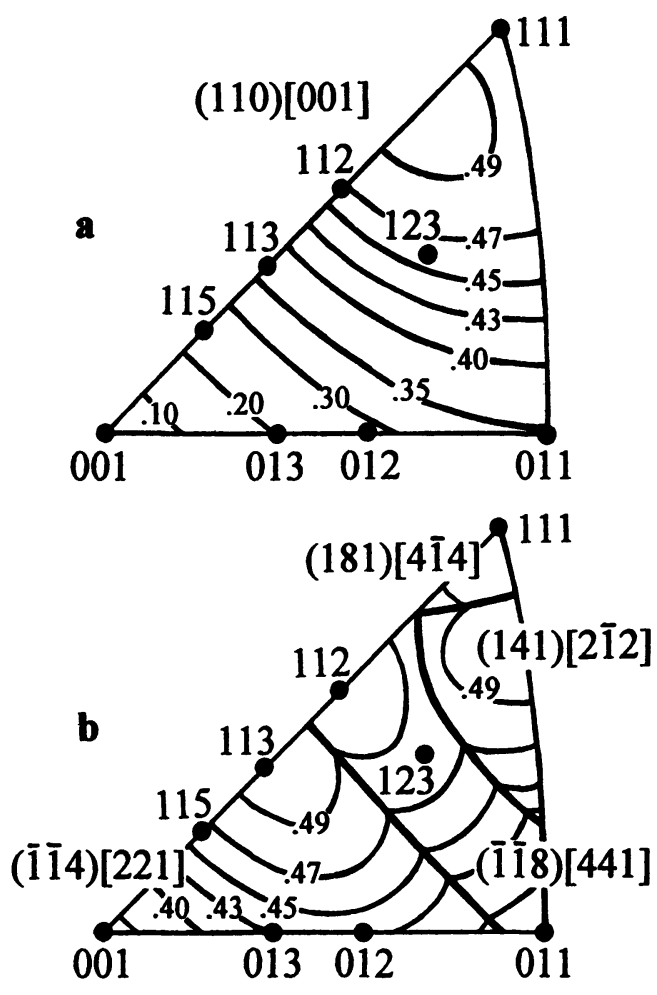

FIGURE 15 Calculated distributions of the Schmid factor for slip systems $\{011\}\langle 001\rangle$ (a) and for twinning systems $\{114\}\langle 221\rangle$ and $\{118\}\langle 441\rangle$ (b). 
obtained results manifest clear evidences of slip in studied single crystals. From this it follows that the critical shear stress for slip by the systems $\{011\}\langle 001\rangle$ is lower, than for both twinning systems.

(5) The above-discussed possibility of twinning in the single crystal with the stable initial orientation $\{111\}\langle 011\rangle(N 19$, Fig. 10) is not contradictory to the statement about the main role of slip in the process of maintenance of the stable orientation. But it seems likely that in this case some competition between slip and twinning takes place because of equally high shear stresses in the systems of slip and twinning.

\subsection{Questions to be Refined}

By the analysis of the obtained experimental data on texture formation in rolled single crystals of $\mathrm{Ti}-\mathrm{Ni}$ we tried to reveal only the most clear evidences of the action of different deformation mechanisms. Though repeated twinning in the studied material is rather probable, it was not considered for the following reasons:

- the used deformation degrees of single crystals with the same orientation are too different to observe, in more detail, successive stages of reorientation (for example, $10 \%$ and $58 \%$ for the initial orientation $\{001\}\langle 011\rangle$ or $10 \%$ and $56 \%$ for the initial orientation $\{011\}\langle 001\rangle)$;

- several twinning cycles with the availability of various twinning systems can result in very different final textures, so that the coincidence of predicted and experimental results would not be a real evidence in favor of the presumed mechanism.

The simplest case of repeated twinning is noted above as applied to the crystal with the initial stable orientation $\{111\}\langle 011\rangle$.

Along with the above-considered variants of texture development, there are two cases of reorientation, having a less clear character from the standpoint of the acting mechanisms:

(a) the initial orientation $\{001\}\langle 011\rangle$ : the transition from the texture of deformation by $10 \%(N 3$, Fig. 2(a) and (b)) to the texture of deformation by $58 \%(N 4$, Fig. 2(c) and (d));

(b) the initial orientation $\{011\}\langle 001\rangle$ : the transition from the texture of deformation by $10 \%$ ( $N$ 6, Fig. 4(a) and (b)) to the texture of deformation by $56 \%(N 7$, Fig. 4(c) and (d)). 
Since in the case (a) the main components of the rolling texture after $\varepsilon_{1}=10 \%$ can be indicated as $\{112\}\langle 111\rangle$ and the main components after $\varepsilon_{2}=58 \%$ as $\{111\}\langle 011\rangle$, this transition is similar to that observed for the crystal with the initial orientation $\{113\}\langle 345\rangle$ after $\varepsilon=43 \%(N 18$, Fig. 9). The orientations $\{112\}\langle 111\rangle$ and $\{113\}\langle 345\rangle$ are close, and therefore it seems likely that their transition to the stable orientation $\{111\}\langle 011\rangle$ would occur in the same manner, i.e. by means of slip or by means of twinning by the system $\{114\}\langle 221\rangle$ followed by slip.

In the case (b) an obvious disagreement of the rolling textures, corresponding to different deformation degrees, can be seen; while after $\varepsilon_{1}=10 \%$ the rolling texture contains a set of mutually symmetric components, the final rolling texture after $\varepsilon_{2}=56 \%$ is asymmetric and consists of the single component $\{111\}\langle 011\rangle$. This disagreement testifies, that plastic deformation of single crystals with the same initial orientation can develop in different manners, bifurcations are possible as for the number of twinning systems, activated at the initial stage of rolling. In order to reveal the concrete way, by which the final texture develops from the intermediate one for the initial orientation $\{011\}\langle 001\rangle$, additional experiments on rolling of single crystals are desirable.

\section{ABOUT INHOMOGENEITY OF PLASTIC DEFORMATION OF SINGLE CRYSTALS}

Manifestations of the inhomogeneous character of plastic deformation in Ti-Ni single crystals are rather numerous. Their features depend on the initial orientation of the crystal as well as on the deformation degree. It is worthwhile to note the following displays of the deformation inhomogeneity, which are of particular interest:

(1) By the deformation degree of 10-15\% essential fractions of crystals with unstable orientations are not yet reoriented, that is they have not yet been deformed, as evidenced by the retention of significant pole density at sites of the initial maxima. But these remainders of initial maxima prove to have very small areas and are situated precisely at the ideal initial orientations. Hence, in single crystals with initial orientations $\{001\}\langle 001\rangle$ and $\{001\}\langle 011\rangle$ (Figs. 1 and 2) those fragments, for which ND was initially noticeably deflected from the normal $\langle 001\rangle$, change their orientation at first. Because of such 
deflection, within these fragments the shear stress for some slip system $\{011\}\langle 001\rangle$ can attain the critical value, resulting in lattice rotation even in the case of unfavorable average orientation of the crystal.

(2) When an initial orientation is symmetric about both RD and TD, as in cases of the initial orientations $\{001\}\langle 001\rangle,\{001\}\langle 011\rangle$ and $\{011\}\langle 001\rangle$, a single crystal "can choose" any of several mutually equivalent variants of reorientation. As a result, the single crystal fragmentates by means of local lattice rotations in opposite directions and ceases to be a single crystal (Figs. 1, 2 and 4).

(3) A similar situation always takes place, when the twinning develops by several planes, as in cases of single crystals with the initial orientations $\{115\}\langle 001\rangle$ and $\{113\}\langle 345\rangle$ (Figs. 3 and 7 ).

(4) Most often, as the deformation degree grows, a single crystal as though "bleeds" from the initial orientation to some intermediate, whereas the scattering of maxima in pole figures increases at the early stage of reorientation and decreases at its following stage. Single crystals, rolled in the initial orientation $\{001\}\langle 011\rangle$ up to different deformation degrees, give a distinct illustration of this phenomenon (Fig. 2): the angular scattering of the texture maxima after $\varepsilon_{1}=10 \%$ is significantly larger, than after $\varepsilon_{2}=58 \%$. Maxima are leaving their initial orientations gradually, by parts, so that at $\varepsilon_{1}=10-15 \%$ the originally compact maxima transform into a complicated distribution extended by several dozens of degrees. But as fragments of the former single crystal approach gradually some intermediate orientation with partial stability, their mutual misorientation decreases. Therefore, in spite of significant orientation scattering at the initial stage of rolling, at the next stage, the former single crystalline state is restored again, at least partially.

An increase of shear stresses does not eliminate the inhomogeneity of plastic deformation in single crystals. The main reason of this inhomogeneity consists in the existence of various systems of slip and twinning with nearly equal values of the Schmid factor in the majority of initial orientations, so that the probabilities of activation of these systems are comparable.

Then the question arises, how regions with different lattice rotations are distributed in space. Two main variants are possible in principle: (a) the single crystal breaks into relatively coarse separate 
macrofragments with different predominant modes of reorientation, so that we may speak about different local textures; (b) microscopic blocks with different orientations are mixed homogeneously within the rolled sample. Since repeated measurements of the same sample give results coinciding very closely and irradiated areas of the sample are rather small, it may be concluded that variant (b) is more probable.

\section{SUMMARY}

(1) Single crystals of the alloy $\mathrm{Ti}-48 \% \mathrm{Ni}-2 \% \mathrm{Fe}$, consisting of the phase $\mathrm{B} 2$, were rolled at $350^{\circ} \mathrm{C}$ with deformation degrees up to $\sim 80 \%$ in 11 different initial orientations. Crystallographic textures of the rolled crystals were studied by means of X-ray diffractometry.

(2) The reorientation modes, chosen by the crystals, as well as the acting mechanisms of plastic deformation depend on the initial orientations of these single crystals.

(3) There are three stable initial orientations, which remain unchanged during rolling of Ti-Ni single crystals: $\{011\}\langle 011\rangle,\{111\}\langle 011\rangle$ and $\{111\}\langle 112\rangle$.

(4) When an initial orientation of the single crystal is unstable, most often it tends to approach the stable orientation $\{111\}\langle 011\rangle$ as the deformation degree increases.

(5) The observed reorientations of the $\mathrm{Ti}-\mathrm{Ni}$ single crystals are consistent with the assumption of the action of the slip systems $\{011\}\langle 001\rangle$.

(6) Twinning makes a significant contribution to plastic deformation of $\mathrm{Ti}-\mathrm{Ni}$ single crystals. It was clearly recognized, that the main twinning planes in the phase B2 are $\{114\}$ and $\{118\}$.

(7) The critical shear stresses of the observed mechanisms of plastic deformation are assumed to increase in the following succession: slip systems $\{011\}\langle 001\rangle$, twinning systems $\{118\}\langle 441\rangle$, twinning systems $\{114\}\langle 221\rangle$.

\section{Acknowledgements}

The authors gratefully acknowledge financial support of the project by the German Research Foundation, DFG. 


\section{References}

Agafonov, V., Legendre, B., Dubertret, A., Rizzo, G. and Dubois, B. (1990). In situ X-ray diffraction observations of phase transitions in $\mathrm{Ti}_{49} \mathrm{Ni}_{51}, \mathrm{Ti}_{49} \mathrm{Ni}_{48} \mathrm{Fe}_{3}$ and $\mathrm{Ti}_{47} \mathrm{Ni}_{51} \mathrm{Zr}_{2}$ alloys as a function of temperature and tensile stress. Materials Science Forum, 56-58, 625-630.

Barrett, C.S. and Massalski, T.B. (1966). Structure of Metals. McGraw-Hill Book Company.

Calnan, E.A. and Clews, C.J.B. (1950). Deformation textures in face-centred cubic metals. Phil. Mag., Ser. 7, 41(322), 1085-1100.

Goo, E., Duerig, T., Melton, K. and Sinclair, R. (1985). Mechanical twinning in $\mathrm{Ti}_{50} \mathrm{Ni}_{47} \mathrm{Fe}_{3}$ and $\mathrm{Ti}_{49} \mathrm{Ni}_{51}$ alloys. Acta metall., 33(9), 1725-1733.

Hoffmann, J., Neff, H., Scholtes, B. and Macherauch, E. (1983). Härterei-Technische Mitteilung, 38, 180.

Moberly, W.J., Proft, J.L., Duerig, T.W. and Sinclair, R. (1990). Deformation, twinning and thermo-mechanical strengthening of $\mathrm{Ti}_{50} \mathrm{Ni}_{47} \mathrm{Fe}_{3}$. Acta metall. mater., 38, 12, 2601-2612.

Perlovich, Yu. (1983). Inhomogeneity of strain hardening, recovery and recrystallization in alloyed molybdenum with the developed crystallographic texture. In: Structure, Texture and Mechanical Properties of Deformed Molybdenum Alloys. Naukova Dumka, Kiev, pp. 87-145.

Perlovich, Yu., Bunge, H.J. and Isaenkova, M. (1997). Inhomogeneous distribution of residual deformation effects in textured BCC metals. Textures \& Microstructures, 29, 241-266.

Shimizu, K. and Tadaki, T. (1987). Shape Memory Alloys, H. Funakubo, Ed., Gordon and Breach Science Publishers.

Vishnyakov, Ya., Babareko, A., Vladimirov, S. and Egiz, I. (1979). Theory of Texture Formation in Metals and Alloys, Nauka, Moscow (in Russian).

Wassermann, G. and Grewen, J. (1962). Texturen metallischer Werkstoffe, SpringerVerlag.

Wcislak, L. and Bunge, H.J. (1996). Texture Analysis with a Position Sensitive Detector, Cuvillier Verlag, Göttingen. 